\title{
A new approach to interventional atrioventricular valve therapy
}

\author{
Andreas Goetzenich, MD, ${ }^{\mathrm{a}, \mathrm{e}}$ Guido Dohmen, MD, ${ }^{\mathrm{a}}$ Nima Hatam, MD,${ }^{\mathrm{a}}$ Thorsten Deichmann, Dipl-Ing, ${ }^{\mathrm{b}}$ \\ Christoph Schmitz, Dipl-Ing, ${ }^{\mathrm{c}}$ Andreas H. Mahnken, MD, PhD, ${ }^{\mathrm{d}}$ Rüdiger Autschbach, MD, PhD, and \\ Jan Spillner, MD
}

\begin{abstract}
Objective: Transcatheter replacement or repair of mitral valve regurgitation has proved demanding. We aimed for a new approach to anchor a biologic heart valve in the mitral position by inserting a valve-carrying hollow body into the left atrium. This approach was investigated in both a simulation and an animal model.
\end{abstract}

\begin{abstract}
Methods: After creating a mold representing the porcine left atrium from the pulmonary veins as far as the mitral valve, a nitinol skeleton was sutured onto interlaced yarns of polyvinylidene flouride fitting the mold. The resulting device was equipped with a commercially available stentless valve $(25 \mathrm{~mm})$ and investigated in a simulator regarding basic functionality. Furthermore, the device was implanted in 8 female pigs through incision of the left atrium during extracorporeal circulation. Before implantation, artificial regurgitation was created by means of excision from the posterior mitral leaflet. Hemodynamic, echocardiographic, and radiologic examinations followed. For a postmortem examination, the entire heart and the lungs were excised.
\end{abstract}

Results: We could demonstrate the functionality of the heart valve in a complex, collapsible, and self-expanding hollow body. The device adapted to the surrounding structures, leading to an exclusion of the left atrium. Sufficient treatment of mitral regurgitation was monitored hemodynamically and by means of echocardiographic analysis, although overall visualization remained difficult. Therefore in 4 animals computed tomographic scans were performed. Autopsy revealed proper positioning without major trauma to the surrounding structures.

Conclusion: Anchoring an additional heart valve in the atrioventricular position does not necessarily need to be performed in the heart valve structure itself. Placement of an additional valve in the mitral position is feasible through this approach. (J Thorac Cardiovasc Surg 2010;140:97-102)

Following basic research in the field of interventional valve therapies, ${ }^{1-4}$ transcatheter implantation of an aortic valve prosthesis has lately become an established method with commercially available prostheses. $^{5-7}$ Also, pulmonary valve placement has been realized. ${ }^{8}$

In western countries mitral regurgitation is one of the most frequent atrioventricular (AV) valve diseases. For many reasons, the state of the art is reconstruction of an $\mathrm{AV}$ valve rather than replacement. One important aspect is preservation of the subvalvular structures, which are essential for ventricular performance. Because interventional mitral valve therapies ${ }^{9-11}$ do not exhibit the same striking results as interventional aortic or pulmonary valve placements, mitral and tricuspid valve therapy mainly remains a task of cardiac surgery. ${ }^{12}$ Interventional placement of a biologic valve in the AV valve position has been marginally investigated.

\footnotetext{
From the Clinics for Cardiothoracic and Vascular Surgery, ${ }^{\mathrm{a}}$ Diagnostic Radiology, ${ }^{\mathrm{d}}$ and Anaesthesiology, ${ }^{\mathrm{e}}$ University Hospital RWTH Aachen; the Institute for Textile Technology (ITA), ${ }^{\mathrm{b}}$ RWTH University Aachen; and Applied Medical Engineering, ${ }^{\mathrm{c}}$ Helmholtz Institute Aachen, Aachen, Germany.

Disclosures: This study has been supported by the HOLSTE-Stiftung, Aachen, Germany.

Received for publication July 7, 2009; revisions received Aug 18, 2009; accepted for publication Sept 17, 2009; available ahead of print Nov 12, 2009.

Address for reprints: Andreas Goetzenich, MD, Department of Anesthesiology, University Hospital RWTH Aachen, Pauwelsstr. 30, D-52074 Aachen, Germany. $0022-5223 / \$ 36.00$

Copyright $(\subset) 2010$ by American Association for Thoracic Surgery

doi:10.1016/j.jtcvs.2009.09.037
}

This is due to the challenging anatomy the mitral valve presents: its annulus and D-shaped circumference lack a sufficient abutment for an annulus-matched anchoring that avoids interference with native valve function or the ventricular outflow tract. In contrast to the emerging technologies for interventional mitral valve repair, our concept was to deliver a self-expanding hollow body carrying a stentless valve prosthesis that would be suspended in the atrium. The mitral valve was chosen to prove our new concept for AV valve therapy, which should be easily adaptable to the tricuspid valve.

Our goal for interventional AV valve therapy was to evaluate the placement of a prosthetic heart valve without the necessity to use the annulus and valve itself for fixation. We aimed to build a prosthesis coating the entire atrial wall from the mitral annulus into the pulmonary veins. This approach would allow the positioning of an additional prosthetic valve in the mitral position, preventing damage to the native valve, the annulus, and the subvalvular apparatus. An additional valve has the theoretic advantage that interventions on the native valve are not necessary, saving the remaining function of the valve. The integrity of the valve-ventricular complex is saved. Furthermore, the additional placement of a valve is probably easier to manage from the hemodynamic point of view than a real replacement because this would imply a time without any functioning AV barrier in a beating heart. An additional effect on leak 


\section{Abbreviations and Acronyms \\ $\mathrm{AV}=$ atrioventricular \\ $\mathrm{CPB}=$ cardiopulmonary bypass}

tightness was expected because of pressure on the annulus and exclusion of the entire left atrium.

To evaluate this approach, we designed several prosthetic prototypes, tested their functionality in vitro, and investigated their performance in a porcine model of mitral insufficiency.

\section{MATERIALS AND METHODS \\ Prosthesis and In Vitro Testing}

The intended prosthesis should cover the entire atrial wall from the pulmonary veins down to the mitral annulus and contain a biologic heart valve (Figure 1, A). We aimed to realize the following requirements: a compressible device with strong self-expansion after release, stability toward compression vertical to the valve plain, shape memory, and low thrombogenicity on the inner surface. In preliminary studies we compared single-wall designs, profiting from a strong self-expanding metallic skeleton to double-walled inflatable models. Although double-walled designs showed smaller sizes in the compressed state, we favored a single-wall design because of the higher stability. The prosthesis hollow body was built of polyvinylidene fluoride with an external nitinol skeleton for stability. Two extensions fit into the pulmonary vein ostia. Molds of the porcine atria allowed adaption to the porcine anatomy. The resulting prototype comprised a stentless valve (SJM Toronto SPV, 25 mm; St Jude Medical, Inc, St Paul, Minn) and nitinol brackets joining the prosthesis' rim to the pulmonary vein stents (Figure 1, B). The load from the valve was displaced through the nitinol skeleton to the atrial wall and the pulmonary vein ostia. Eight such prostheses were manufactured in cooperation with the Institute for Textile Technology.

All prostheses were first observed and tested in the pulsatile circulatory mock-loop simulator at the Aachen Helmholtz Institute. For this cause, an elastic silicone model of the porcine left atrium, including the pulmonary veins and the upper part of the mitral valve, was generated. Observation of the prostheses' behavior and valve function inside the model atrium was facilitated by the model's opacity. The prostheses were compressed, inserted through the mitral valve part of the model atrium, and released again. Thereafter, the model was connected to the simulator with an inlet through the pulmonary veins and outflow through the mitral valve. Pulsatile circulation was established and continued for 180 minutes. The circulatory mockloop simulator worked at heart rates of 70 up to 110 beats $/ \mathrm{min}$ and at cardiac outputs of 2, 4, 5, and $7 \mathrm{~L} / \mathrm{min}$, respectively (US Food and Drug Administration requirements). It was filled with saline. Positioning of the hollow body, its stability, and the function of the embedded valve prosthesis were monitored.

\footnotetext{
Animal Model

Eight female pigs of $70 \mathrm{~kg}$ body weight were used for the animal investigations. The animal research protocol was approved by the local authorities (9.93.2.10.35.07.013). The experimental animals received humane care in accordance with the applicable National Institutes of Health publication (no. 86-23, revised 1985). Routine monitoring and catheterization for cardiothoracic surgery was performed. All animals were killed humanely after completion of the investigation. After premedication, general anesthesia was induced, followed by baseline measurement of hemodynamics. Double peripheral venous cannulation (25F, Biomedicus; Medtronic, Minneapolis,
}

Minn) was performed, followed by a left thoracotomy in the sixth intracostal space. An arterial cannula (22F, DLP) was inserted and fixed to the descending aorta. The pericardium was opened, and the left atrium was exposed. After therapeutic heparinization, normothermic full cardiopulmonary bypass (CPB) was established. The following procedures were performed on the beating heart. The left atrium was longitudinally incised for about $5 \mathrm{~cm}$. The native mitral valve was then inspected, and an artificial insufficiency was created by a $0.5 \mathrm{~cm}^{2}$ excision from the posterior valve leaflet. The size of the left atrium was compared with that of the manufactured prostheses, and the right size was chosen by means of visual inspection.

The crimped prosthesis was inserted through the atrial incision. First, the pulmonary vein stents were placed into the ostia, and the stents were expanded. Then the remaining prosthesis with the valve was placed above the mitral annulus, and the hollow body was released. After deairing, the left atrium was closed with sutures. The animal was slowly weaned from $\mathrm{CPB}$ and decannulated. Coagulation was reconstituted with protamine to an activated clotting time of less than 120 seconds.

Repeated epicardial, transthoracic, and transesophageal echocardiographic analysis (GE Vivid 5; GE Healthcare, Waukesha, Wis) was performed, as well as postinterventional hemodynamic measurements (Datex-Ohmeda, GE Healthcare) and radiologic investigations (fluoroscopy and computed tomography; Siemens, Berlin, Germany).

The animals were killed after 90 minutes of postoperative observation and investigation and 60 minutes after administration of protamine. For postmortem examination, the heart was carefully excised with adjacent lungs. Both the lung veins and the left ventricle were opened before the left atrium to completely check positioning. After careful washing with saline, the prostheses were studied to identify clot formation.

\section{RESULTS \\ Prosthesis In Vitro Testing}

Before implantation, all prostheses were investigated in the circulatory simulator. The construction method of the simulator together with the transparent model atrium allowed a good assessment of the prosthesis' overall performance (Figure 2). Also, the implantation procedure of the prosthesis could be controlled by means of visual inspection. A simple technique was developed to compress and release the 2 extensions and the main body independently. For implantation, the completely compressed prosthesis had a dimension of about $1.5 \times 9.5 \mathrm{~cm}$. After insertion of the prosthesis into the atrium, the 2 venous extensions were released one after the other, followed by the release of the main body. All prostheses fitted the model atria very well. There were no dislodgements of the expanded prosthesis, even at a maximal circulation of $7 \mathrm{~L} / \mathrm{min}$. The prosthesis laid tightly on the model annulus in all cases. The valve re-expanded inside the prosthesis, and there was no visual evidence for stenosis or insufficiency. In one case the suture fixation between the prosthesis body and the prosthetic valve became insufficient over time. This problem was solved by resuturing.

\section{Animal Experiments}

All 8 animals survived the operation, CPB time, and the follow-up of 90 minutes without CPB. The prostheses could be surgically implanted through the longitudinal atriotomy 

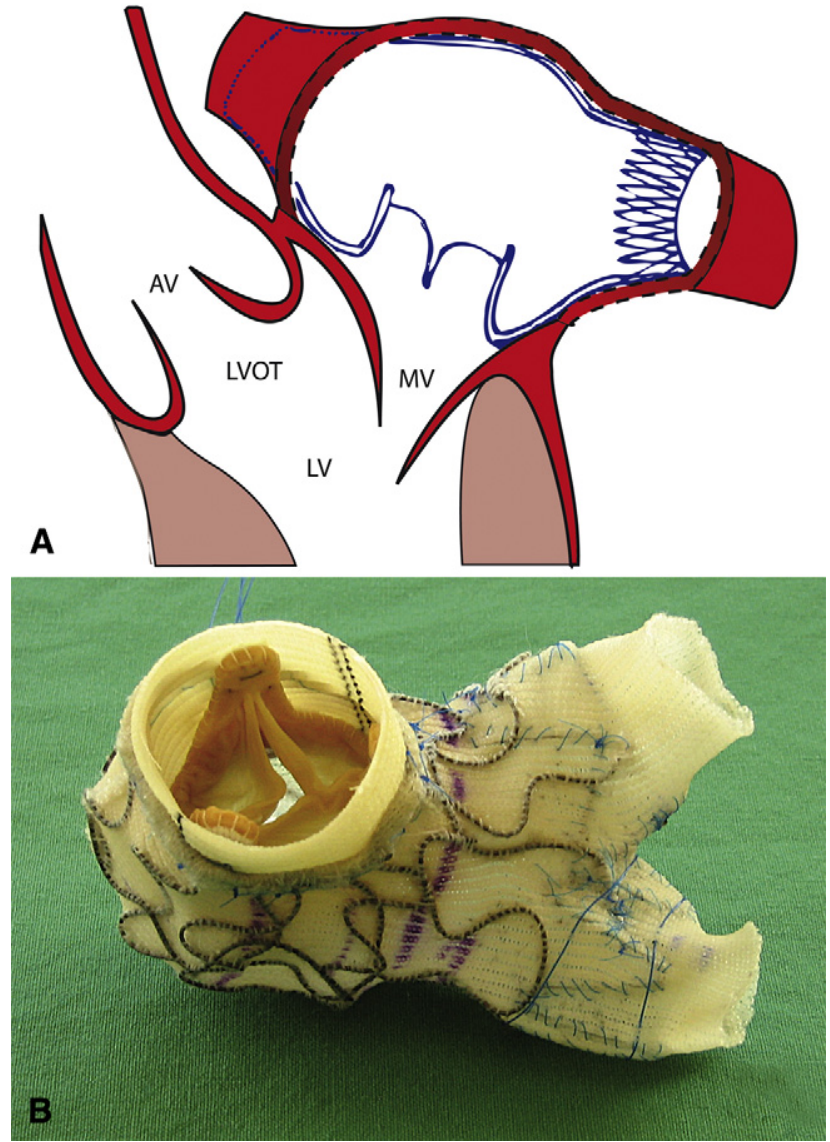

FIGURE 1. Schematic drawing of a prosthesis (blue) in the left porcine atrium with 2 pulmonary veins (A) and an early prototype of the prosthesis with an integrated valve (B). $A V$, Aortic valve; $L V O T$, left ventricular outflow tract; $L V$, left ventricle; $M V$, mitral valve.

in all animals. Tight closure of the fragile atrial wall was sometimes challenging. In one case atrial fibrillation occurred during implantation and was successfully treated with electrolytes and a single shot of $300 \mathrm{mg}$ of amiodarone. After termination of CPB and a brief compensatory period, hemodynamics reached a steady state comparable with preprocedural conditions in all animals. In particular, there was no evidence for a significant pulmonary hypertension or a $\mathrm{V}$ wave in the wedge pressure recording.

Echocardiography turned out to be very difficult, probably because of the amount of foreign material inside the heart. Although many acoustic windows were tried in each animal, only limited echocardiographic access to the valve prosthesis was possible. The prosthesis showed good adaptation in the region of the mitral annulus, and no relevant movement of the prosthesis was detectable. Both the native and the prosthetic mitral valve moved synchronously (Figure 3).

There was no evidence for left ventricular outflow tract obstruction or deformation of the aortic root in any case. Although a higher-grade mitral regurgitation was seen at the

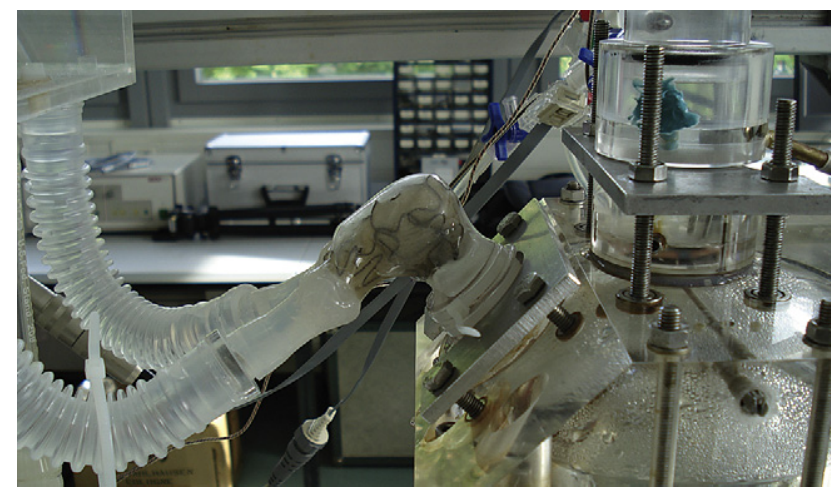

FIGURE 2. Prosthesis prototype in a circulatory mock-loop simulator.

posterior leaflet of the native valve (artificial defect), the insufficiency jet was stopped at the additional valve. The valve prosthesis itself appeared with a normal movement of the cusps and without regurgitation or stenosis. Echocardiographically, it was not possible to gain information about paravalvular leakage or adherence to the atrial wall.

Fluoroscopic analysis was performed both as angiographic analysis through the pulmonary artery and ventriculographic analysis from the apex of the left ventricle. These radiographic investigations demonstrated full patency of the pulmonary veins into the atrium and straight passage of the contrast medium into the ventricle. No relevant regurgitation over the prosthetic valve could be detected. A slight deposit of contrast medium was seen in the area of the anterior mitral leaflet between the atrial wall and the prosthesis. After restoration of coagulation capacity, no relevant hemodynamic changes indicating severe clot formation or valve thrombosis occurred.

As a result of difficult visualization, the last 4 animals were subjected to a computed tomographic scan. In this investigation the intended proper position of the prosthesis in the left atrium could be verified (Figure 4). Nevertheless, accurate information about paraprosthetic leakage was still not possible.

Autopsy revealed a correct (intended) overall position of the prosthesis in all cases. The prosthesis body was pressed onto the mitral valve annulus, and in 3 animals a slight gliding of the prosthesis into the mitral valve was seen. Except for one case of a dislodged skeletal strut, there was no trauma to intracardiac structures. No relevant restriction of the mitral valve leaflets and no left ventricular outflow tract obstruction could be detected (Figure 5, A). In one case improper release of the prosthesis extension led to an occlusion of the right pulmonary vein. In another case a small tear in the right pulmonary vein caused by a sharp dislodged strut had to be recognized. In all other animals, the extensions of the prosthesis could be found in a proper position in the pulmonary vein ostia, sufficiently draining the pulmonary veins (Figure 5, B). No relevant thrombus was found inside the entire prosthesis. 

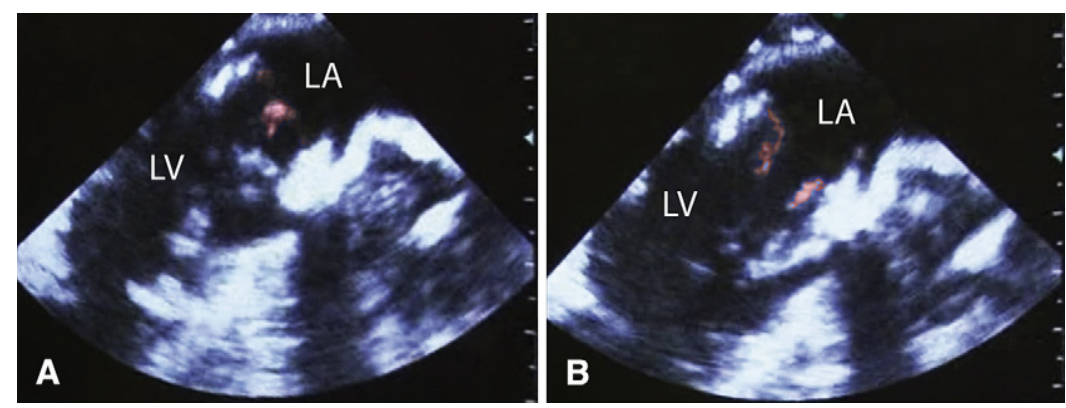

FIGURE 3. Epicardial echocardiographic image of the mitral valve with an additional prosthesis (red) in the closed (A) and opened (B) state. $L V$, Left ventricle; $L A$, left atrium.

\section{DISCUSSION}

Surgical repair is by far the most successful treatment for mitral valve disease. Nevertheless, in the last decade, interventional treatment of valve diseases emerged for highrisk patients, with strikingly good results, especially in the treatment of aortic stenosis. For AV valves, especially for the mitral valve, several interventional approaches to treat insufficiencies were proposed, ${ }^{9}$ and some of them are still on the bench. In contrast to the results in interventional aortic valve treatment, clinical results for the repair of AV valves have not been that striking. Mainly, second-choice surgical techniques are translated into interventional techniques, such as the Alfieri stitch being translated into the Evalve MitraClip with thus far limited success. Yet all these techniques are still being improved, and further approaches to beatingheart valve therapy are investigated. ${ }^{13,14}$

The interventional placement of a prosthetic valve in the $\mathrm{AV}$ position is rarely investigated because the issue of anchoring is very challenging. ${ }^{15}$ The $\mathrm{AV}$ valve structure is not tubular and, therefore, a simple stent cannot be placed. Furthermore, the valve and its annulus are an integrated part of contraction and ventricular function. Movement of the leaflets also provides sustained forward flow of the blood.
Our concept allowed us to show that it is indeed possible to place an additional valve without compromising the native valvular function of both the aortic and mitral valves. The angled AV passage makes it very demanding to find the right site for anchoring a foreign body without danger to the surrounding structures. It is particularly significant to avoid outflow tract obstruction that could occur in the case of an intravalvular placement of a prosthetic valve. Our approach was to spread the force transduction as evenly as possible over the entire atrial wall up to the pulmonary vein ostia to avoid protrusion and the resulting compression to surrounding structures. We did not monitor any harm to the outflow tract or aortic valve function and demonstrated normal movement of the remaining mitral cusps.

The principle to exclude a pathology from blood flow was one of the first purposes of interventional therapies. Interventional exclusion of aortic aneurysms has shown excellent results. Nevertheless, apart from the exclusion of the left atrial appendage to prevent strokes, ${ }^{16}$ no further exclusions of parts of the heart had been investigated thus far. In our investigation an exclusion of the whole left atrium was intended as a part of valve fixation. We demonstrated that insertion of a through-flown hollow body to exclude a heart chamber is feasible and does not lead to hemodynamic
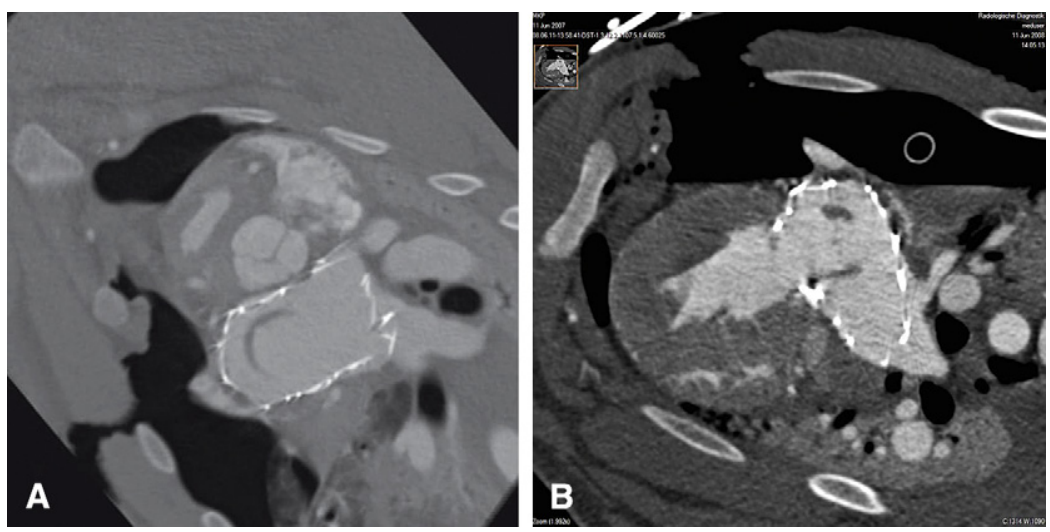

FIGURE 4. Computed tomogram showing a prosthesis in the valve plane (A) and in a slightly oblique longitudinal axis (B). 

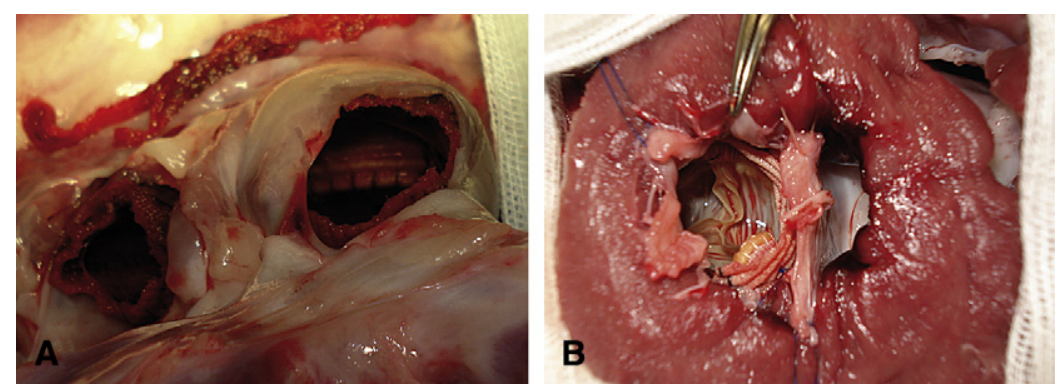

FIGURE 5. Autopsy specimen with a view from the apex showing the prosthetic mitral valve and unobstructed outflow tract (A) and drainage of both pulmonary veins (B).

depression. Even though we detected a slight dehiscence of the prosthesis body from the atrial wall in the area of the anterior mitral leaflet, there was no continuous extra-prosthetic blood flow reaching the pulmonary veins. It is assumed that in the long run these wake zones will clot and provide additional tightness. The lost atrial contraction seems not to be relevant in $\mathrm{AV}$ valve diseases because atrial contraction is already compromised in many cases as a result of atrial fibrillation and dilatation. In contrast, the relevance of a large foreign surface inside the hollow body regarding thrombosis cannot be judged by our investigations because long-term experiments would be needed. Chronic anticoagulation will surely be needed.

\section{Complexity of Transfer to Interventional Application in Patients}

Our work investigated the principal feasibility of anchoring a biologic heart valve in the AV position by inserting a valve-carrying hollow body into the atrium. The preliminary design of our prosthesis led to minor technical problems, such as the slight dehiscence of the prosthesis in the area of the anterior mitral leaflet or fracture of the nitinol struts. A more professional design should be able to overcome these flaws. Although we constructed a collapsible self-expanding prosthesis, we chose an open surgical access with CPB for insertion. A minimally invasive or transcatheter placement was put on hold because the major principal questions of such an approach had to be cleared. In its current form, a transapical implantation seems favorable because the current body size exceeds the limits for a transseptal approach. Additionally, the transvalvular entry into the atrium facilitates the cannulation of the pulmonary veins. Nevertheless, these limitations might be overcome by future designs. Currently, the idea of a stepwise implantation is under construction. This includes valve deployment after insertion of a prosthesis body into the atrium.

In our experiments we used the pig because of its very well-suited anatomy with respect to the pulmonary veins. In human subjects the anatomy of the atrium seems to be much more demanding for an exclusion because of both variable size and inflow of 4 pulmonary veins. Adaptation to each patient would require an individual sizing process, including imaging and measurement of the native atrium and pulmonary ostia, followed by selection of an appropriately sized or even a personalized fabrication of the hollow body, a process modern production technology is able to provide (rapid prototyping).

\section{LIMITATIONS}

Because our treatment of valvular disease favors a completely new approach to the subject, our study was planned as a proof of concept. Therefore all midterm and long-term results will have to be tested in further experiments. These will first of all have to dispel concerns about long-term stability and chronic cardiac complications.

\section{CONCLUSION}

We demonstrated the principal feasibility of a new approach to additionally place a heart valve in the AV position in a complex collapsible and self-expanding hollow body. The new type of prosthesis led to an exclusion of a complete heart chamber. Further work based on this concept seems promising and is on the way. Technical refinements and long-term experiments are needed for terminal judging of this approach.

\section{References}

1. Andersen HR, Knudsen LL, Hasenkamm JM. Transluminal implantation of artificial heart valves. Description of a new expandable aortic valve and initial results with implantation by catheter technique in closed chest pigs. Eur Heart J. 1992; 13:704-8.

2. Bonhoeffer P, Boudjemline Y, Qureshi SA, et al. Percutaneous insertion of the pulmonary valve. J Am Coll Cardiol. 2002;39:1664-9.

3. Cribier A, Eltchaninoff $\mathrm{H}$, Bash A, et al. Percutaneous transcatheter implantation of an aortic valve prosthesis for calcific aortic stenosis: first human case description. Circulation. 2002;106:3006-8.

4. Spillner J, Börgermann J, Reppenhagen G, Er-Xiong L, Reidemeister JC Friedrich I. An experimental approach to reversible "endovascular aortic valve placement." J Heart Valve Dis. 2005;14:546-50.

5. Cribier A, Eltchaninoff H, Tron C, et al. Treatment of calcific aortic stenosis with the percutaneous heart valve: mid-term follow-up from the initial feasibility studies: the French experience. J Am Coll Cardiol. 2006;47:1214-23.

6. Grube E, Laborde JC, Gerckens U, et al. Percutaneous implantation of the CoreValve self-expanding valve prosthesis in high-risk patients with aortic valve disease: the Siegburg first-in-man study. Circulation. 2006;114 1616-24. 
7. Walther T, Simon P, Dewey $\mathrm{T}$, et al. Transapical minimally invasive aortic valve implantation: multicenter experience. Circulation. 2007;116(suppl): I240-5.

8. Khambadkone $S$, Bonhoeffer P. Nonsurgical pulmonary valve replacement: why, when, and how? Catheter Cardiovasc Interv. 2004;62:401-8.

9. Mack MJ. New techniques for percutaneous repair of the mitral valve. Heart Fail Rev. 2006;11:259-68.

10. Herrmann HC, Rohatgi S, Wasserman HS, et al. Mitral valve hemodynamic effects of percutaneous edge-to-edge repair with the MitraClip device for mitral regurgitation. Catheter Cardiovasc Interv. 2006;68:821-8.

11. Silvestry FE, Rodriguez LL, Herrmann HC, et al. Echocardiographic guidance and assessment of percutaneous repair for mitral regurgitation with the Evalve $\mathrm{Mi}$ traClip: lessons learned from EVEREST I. J Am Soc Echocardiogr. 2007;20: 1131-40.
12. Block PC. Percutaneous mitral valve repair: are they changing the guard? Circulation. 2005;111:2154-6.

13. Rosengart TK, Feldman T, Borger MA, et al. Percutaneous and minimally invasive valve procedures: a scientific statement from the American Heart Association. Circulation. 2008;117:1750-67.

14. Bajona P, Katz WE, Daly RC, Zehr KJ, Speziali G. Beating-heart, off-pump mitral valve repair by implantation of artificial chordae tendineae: an acute in vivo animal study. J Thorac Cardiovasc Surg. 2009;137:188-93.

15. Ma L, Tozzi P, Huber CH, Taub S, Gerelle G, von Segesser LK. Double-crowned valved stents for off-pump mitral valve replacement. Eur J Cardiothorac Surg. 2005;28:194-9.

16. Nakai T, Lesh MD, Gerstenfeld EP, Virmani R, Jones R, Lee RJ. Percutaneous left atrial appendage occlusion (PLAATO) for preventing cardioembolism: first experience in canine model. Circulation. 2002;105:2217-22. 